\title{
A Sequential Conic Programming Approach for the Coordinated and Robust Design of Power System Stabilizers
}

\author{
Rabih A. Jabr, Member, IEEE, Bikash C. Pal, Senior Member, IEEE, and \\ Nelson Martins, Fellow, IEEE
}

\begin{abstract}
This paper shows that conic programming is an effective tool to solve robust power system stabilizer (PSS) design problems, namely coordinated gain tuning and coordinated phase and gain tuning. Design robustness is achieved by simultaneously considering several operating scenarios. The method is implemented through a sequence of conic programming runs that define a multivariable root locus along which the eigenvalues move. Specifically, the eigenvalues corresponding to the unstable and poorly damped modes are moved to a conic sector in the left half of the s-plane, whereas the eigenvalues corresponding to the well damped modes are constrained to stay within the boundaries of this conic sector. At each step of the solution, the PSS design parameters are restricted in a trust-region such that the computation of the eigenvalue shift based on the residue method holds valid. The proposed method is demonstrated on a 68-bus test system with 9 different operating conditions. Comparisons are carried out between conic programming implementations for PSS coordinated gain tuning and for simultaneous tuning of gain and phase characteristics.
\end{abstract}

Index Terms-- Controller design, coordinated design, multivariable systems, nonlinear programming, optimization methods, power system stabilizers, robust control, small-signal stability.

\section{NOMENCLATURE}

$F_{k}(s)$ Block of the $k^{\text {th }}$ PSS transfer function with unknown design parameters

$G_{k}(s)$ Transfer function of the $k^{\text {th }}$ PSS with unity gain

$H_{k}(s)$ Transfer function of the $k^{\text {th }}$ PSS

$H_{k i}(s) i^{\text {th }}$ component in the summation of $H_{k}(s)$

$\varphi_{k}(s)$ Torsional filter block of the $k^{\text {th }}$ PSS

$N_{a} \quad$ Number of unstable and poorly damped modes

$N_{b} \quad$ Number of well damped modes

$N_{c} \quad$ Number of cases or operating scenarios

R. A. Jabr is with the Electrical, Computer and Communication Engineering Department, Notre Dame University, P. O. Box 72 Zouk Mikayel, Zouk Mosbeh, Lebanon (e-mail: rjabr@ndu.edu.lb).

B. C. Pal is with the Electrical and Electronic Engineering Department, Imperial College, London SW7 2AZ, U.K. (e-mail: b.pal@imperial.ac.uk).

N. Martins is with CEPEL, 21941-911 Rio de Janeiro, RJ, Brazil (e-mail: nelson@cepel.br).

\author{
$N_{S} \quad$ Number of PSSs \\ $r_{j k} \quad$ Residue associated with the $j^{\text {th }}$ eigenvalue and $k^{\text {th }}$ PSS \\ $s \quad$ Complex frequency \\ $T_{k} \quad$ Lag time constant of the $k^{\text {th }}$ PSS \\ $T_{w} \quad$ Washout time constant \\ $t_{j} \quad$ Euclidean distance of the $j^{\text {th }}$ eigenvalue shift \\ $t_{R j} \quad$ Projection of $t_{j}$ on the real axis \\ $t_{l j} \quad$ Projection of $t_{j}$ on the imaginary axis \\ $u_{k}(t)$ Output of the $k^{\text {th }}$ PSS as a function of time \\ $x_{k i} \quad i^{\text {th }}$ design parameter of the $k^{\text {th }}$ PSS \\ $\mathfrak{R}($.$) \quad Real part of a complex number$ \\ $\Im$ (.) Imaginary part of a complex number \\ $\lambda_{j} \quad j^{\text {th }}$ eigenvalue with nonnegative imaginary part \\ $=-\alpha_{j}+i^{\prime} \omega_{j}$ \\ $\rho \quad$ Trust-region limit \\ $\varsigma$ Boundary damping ratio $=\cos \theta$ \\ $\varsigma^{\prime} \quad$ Target damping ratio $=\cos \theta^{\prime}$ \\ $\Delta \omega_{k}(t)$ Speed deviation of the $k^{\text {th }}$ machine as a function of \\ time \\ $\Delta \quad$ Incremental change in a quantity \\ c Superscript/subscript denoting the case number \\ o/* Superscript denoting the current/optimal value \\ - $\quad$ Normalized value of a quantity
}

\section{INTRODUCTION}

$\mathrm{P}$ OWER system stabilizers are the most cost effective controls for damping electromechanical oscillations [1]. They act through the generator's voltage regulator and ideally produce a component of electrical torque on the rotor which is in phase with speed deviations over a wide range of frequencies of oscillation. The basic structure of the PSS comprises a gain, phase compensation blocks, a washout filter, and output limiters. With speed or frequency used as PSS inputs, a torsional filter is also commonly used. The PSS design problem therefore calls for coordinating the parameters of the different stabilizers so that the damping of the system's electromechanical modes is increased. An important issue in the design of controllers is robustness, i.e. the controller 
should achieve the desired damping over a wide range of system operating conditions.

The power systems literature reports several methods for PSS tuning and coordination with electronic controls such as flexible AC transmission system (FACTS) devices. The gain and phase compensation approach [2] is one of the most widely adopted methods for designing system controls. Reference [3] shows that for a PSS with speed input, the phase compensation block should be designed to compensate the phase lag between the automatic voltage regulator reference voltage and the electrical torque of the generator with the generator angle held constant. Early research has suggested that the classical gain and phase compensation approach is applicable to robust PSS design. This is based on the observation that the torque-reference automatic voltage regulator transfer function remains essentially invariant over a wide range of conditions encountered in the operation of the system [4]. The gains of the different controllers are subsequently chosen to achieve the desired damping level. The coordinated gain tuning method has been recently demonstrated using linear programming [5], [6], NewtonRaphson method [7], and nonlinear least squares based multivariable root following technique for multiple operating scenarios [8]. References [9] and [10] show that linear and quadratic programming approaches can be employed for the coordinated gain and phase tuning of the various PSSs. In [9], [10] the objective has been set to minimize the summation of controllers' magnitudes at typical frequencies of oscillation. Robustness in controller design has been also approached through the normalized eigenvalue distance minimization method [11], [12]. This method maintains acceptable performance over different operating conditions by minimizing the maximum normalized distance. Another technique to obtain stability robustness is through norm optimization formulations that are minimized using algorithms for linear matrix inequalities [13]. Search based methods, such as genetic algorithms [14], [15], [16], have been also proposed for robust PSS design. A comprehensive review of coordinated PSS design methods appears in [17].

Although PSS tuning is primarily targeted at damping electromechanical modes, the arbitrary setting of eigenvalue locations corresponding to these modes may adversely affect the system's control modes [15]. This paper proposes an approach for tuning PSS parameters such that both the electromechanical and control modes are well damped. It is applicable to both coordinated PSS gain and coordinated PSS gain and phase tuning. The approach incrementally shifts the eigenvalues corresponding to the unstable and poorly damped modes to a conic sector in the left half of the s-plane whilst prohibiting the eigenvalues originally in this sector from leaving it. The coordination of the eigenvalue movement is achieved via a conic programming method that successively reduces the distance between the current and optimal eigenvalue locations. At each iteration, the conic program is formulated based on residues that give the sensitivity of an eigenvalue due to feedback with a PSS transfer function [1].
The validity of the eigenvalue shifting approximation as predicted by the sensitivity information is maintained throughout the solution process by employing a trust-region technique, i.e. the PSS parameter increments are restricted in a region such that the sensitivity information remains valid. The controller robustness is addressed by simultaneously considering, within the conic quadratic programming framework, the eigenvalue locations corresponding to scenarios under normal conditions or contingencies. Although this increases the size of the conic quadratic program, a solution can still be efficiently obtained. In fact, conic quadratic problems are convex and can be solved by polynomial-time interior-point methods at basically the same computational complexity as linear programming problems of similar size [18].

The rest of this paper is organized as follows. Section II presents the motivation of the method as an optimization based multivariable root locus following technique [19]. Section III reviews the shift of eigenvalues as a function of the PSS parameter changes. The proposed conic programming approach for coordinated gain tuning is introduced in section IV. The extension of the method for coordinated gain and phase tuning is outlined in section V. Section VI includes numerical results on the 68-bus test system. The paper is concluded in section VII with directions for future research.

\section{Motivation: Multivariable Root Locus Following TECHNIQUE}

The proposed method is motivated by the root locus approach, which is one of the most intuitive techniques for controller coordinated gain tuning. Assume that the power system dynamic model for a given operating condition has been put into state-space form. With the PSS phase characteristic tuned to approximately match the ideal phase lead, the root locus for a specific PSS is used to identify the gain that gives sufficient damping to the modes of interest. The stabilizers are designed sequentially, i.e. a designed PSS is treated as the only control loop that is open in a multivariable system in which all other PSSs are operating in closed loop. Because a PSS may increase the stability of some modes at the expense of reducing the damping of others, some stabilizers may require more than one pass through the tuning algorithm to reach a compromise between the design parameter values.

If $\lambda_{j}^{0}$ is an eigenvalue on the root locus, then the movement of this eigenvalue due to a sufficiently small change in the PSS gain can be approximated by the movement along the straight line tangent to the locus at $\lambda_{j}^{0}$ [20]. The slope of this tangent line can be derived from residue information [1]. In fact, the sensitivity information is not limited to the change of one PSS gain, but can be generalized to simultaneously include different controllers. The aim of this work is to propose a sequential conic programming method to incrementally adjust the PSS gains. This can be done by coordinating the movement of the eigenvalues that define different generalized root loci corresponding to different operating conditions. The 
generalized root loci are dependent on the design method [19]. The conic program uses sensitivity information and Euclidean distance minimization to direct the generalized root loci into satisfactory regions of the eigenvalue space. An extension for coordinated tuning of both the phase leads and gains of the PSSs is also proposed. Related work appears in [5]-[10].

\section{EIgENVALUe Sensitivity to DyNAmic FeEdBack}

Let $H_{k}(s)$ for $k=1, \ldots, N_{S}$ denote the transfer function of the $k^{\text {th }}$ PSS with speed input

$$
\begin{aligned}
H_{k}(s) & =F_{k}(s) \frac{s T_{w}}{1+s T_{w}} \varphi_{k}(s) \\
& =\frac{x_{k 2} s^{2}+x_{k 1} s+x_{k 0}}{\left(1+s T_{k}\right)^{2}} \frac{s T_{w}}{1+s T_{w}} \varphi_{k}(s)
\end{aligned}
$$

where $\left(x_{k 0}, x_{k 1}, x_{k 2}\right)$ are the parameters of the $k^{\text {th }}$ PSS to be tuned, $T_{k}$ is a given PSS lag time constant, $T_{w}$ is the washout time constant, $\varphi_{k}(s)$ is the torsional filter transfer function if available, and $N_{S}$ is the number of stabilizers. Equation (1) can be rewritten as

$$
H_{k}(s)=\sum_{i=0}^{2} x_{k i} H_{k i}(s)
$$

where

$$
\begin{aligned}
& H_{k 0}(s)=\frac{1}{\left(1+s T_{k}\right)^{2}} \frac{s T_{w}}{1+s T_{w}} \varphi_{k}(s) \\
& H_{k 1}(s)=s H_{k 0}(s) \\
& H_{k 2}(s)=s^{2} H_{k 0}(s) .
\end{aligned}
$$

The shift in the $j^{\text {th }}$ eigenvalue $\lambda_{j}$ due to its sensitivity to dynamic feedback through the PSS transfer functions in (1) is given by [1], [17]

$$
\lambda_{j}=\lambda_{j}^{0}+\sum_{k=1}^{N_{s}} \sum_{i=0}^{2} \Delta x_{k i} r_{j k} H_{k i}\left(\lambda_{j}^{0}\right)
$$

where

$\lambda_{j}^{0}=$ current location of the $j^{\text {th }}$ eigenvalue

$r_{j k}=$ residue associated with the $j^{\text {th }}$ eigenvalue and $k^{\text {th }}$ PSS

$\Delta x_{k i}=x_{k i}-x_{k i}^{0}=$ incremental change in PSS parameters.

Equation (6) is applicable for the simultaneous tuning of the

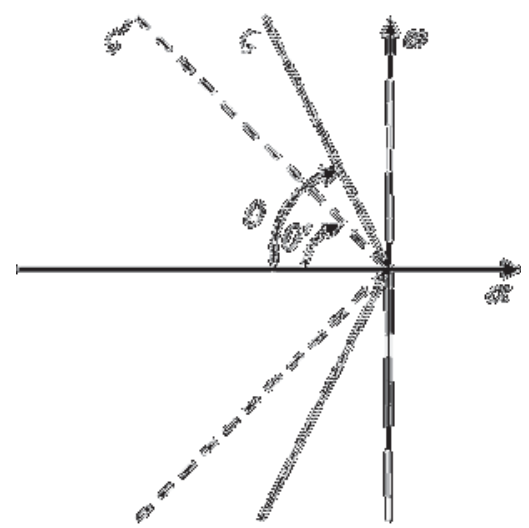

PSS gain and phase. In the gain coordination approach, the phase lead characteristic is specified independently and (6) reduces to

$\lambda_{j}=\lambda_{j}^{0}+\sum_{k=1}^{N_{s}} \Delta x_{k 0} r_{j k} G_{k}\left(\lambda_{j}^{0}\right)$

where $H_{k}(s)=x_{k 0} G_{k}(s)$.

\section{Conic Programming Approach For CoORdinated GAIN TUNING}

\section{A. Incremental Eigenvalue Shifting}

Let $\varsigma=\cos \theta$ denote the damping ratio corresponding to the boundaries of the conic sector in Fig. 1. A mode whose damping ratio is less than $\varsigma$ can have its eigenvalue incrementally moved into the conic sector by adjusting the PSS gain $x_{k 0}$ to successively reduce the Euclidean distance between the original and optimal eigenvalue locations in the splane. Let $\lambda_{j}$ denote an eigenvalue with nonnegative imaginary part corresponding to a mode whose damping is to be improved. The incremental Euclidean distance minimization can be cast as the following quadratic cone program

minimize $t_{j}$ subject to

$$
\begin{aligned}
& t_{j} \geq \sqrt{t_{R j}^{2}+t_{l j}^{2}} \\
& t_{R j}=\Re\left(\lambda_{j}^{*}-\lambda_{j}^{0}\right)-\sum_{k=1}^{N_{S}} \Re\left(r_{j k} G_{k}\left(\lambda_{j}^{0}\right)\right) \Delta x_{k 0} \\
& t_{l j}=\Im\left(\lambda_{j}^{*}-\lambda_{j}^{0}\right)-\sum_{k=1}^{N_{s}} \Im\left(r_{j k} G_{k}\left(\lambda_{j}^{0}\right)\right) \Delta x_{k 0} \\
& x_{k 0}^{0}+\Delta x_{k 0} \geq 0,\left|\Delta x_{k 0}\right| \leq \rho, k=1, \ldots, N_{s}
\end{aligned}
$$

where $\mathfrak{R}($.$) and \mathfrak{I}($.$) respectively denote the operators that$ yield the real and imaginary parts of a complex quantity, $\rho$ is the trust-region limit, and $\lambda_{j}^{*}=-\alpha_{j}^{*}+i^{\prime} \omega_{j}^{*}$ is the optimal eigenvalue ( $i^{\prime}$ is the complex imaginary unit). The real part of $\lambda_{j}^{*},-\alpha_{j}^{*}$, is a design variable and the imaginary part is

$$
\omega_{j}^{*}=\tan \left(\cos ^{-1} \varsigma^{\prime}\right) \alpha_{j}^{*}, \quad \alpha_{j}^{*} \geq 0 .
$$

The target damping ratio $\varsigma^{\prime}$ is chosen to be slightly greater than $\varsigma$ so that whilst approaching this target, the eigenvalue will end up being inside the conic sector of damping ratio $\varsigma$. The specification of the optimal eigenvalue in the vicinity of the conic sector boundary ensures that the eigenvalue travels a minimum distance on the generalized root locus to be located within the conic sector. Based on the current residue information, the solution of the quadratic conic program yields the PSS parameters that produce an incremental movement of the eigenvalue along this locus.

In practice, $N_{a}$ different unstable and poorly damped modes need to have their damping improved. The objective would therefore require minimizing the sum of eigenvalue Euclidean distances 
minimize $\sum_{j=1}^{N_{a}} t_{j}$ subject to

$$
\text { (10)-(12), (14) for } j=1, \ldots, N_{a} \text { and (13). }
$$

In addition, throughout the eigenvalue shifting process, the well-damped modes are constrained not to leave the conic sector by enforcing the following constraints for $j=1, \ldots, N_{b}$, where $N_{b}$ is the number of well damped modes

$$
\begin{aligned}
& \mathfrak{I}\left(\lambda_{j}^{0}\right)+ \sum_{k=1}^{N_{S}} \mathfrak{I}\left(r_{j k} G_{k}\left(\lambda_{j}^{0}\right)\right) \Delta x_{k 0} \leq \\
&-\tan \theta\left[\mathfrak{R}\left(\lambda_{j}^{0}\right)+\sum_{k=1}^{N_{S}} \mathfrak{R}\left(r_{j k} G_{k}\left(\lambda_{j}^{0}\right)\right) \Delta x_{k 0}\right] \\
& \mathfrak{I}\left(\lambda_{j}^{0}\right)+\sum_{k=1}^{N_{S}} \mathfrak{I}\left(r_{j k} G_{k}\left(\lambda_{j}^{0}\right)\right) \Delta x_{k 0} \geq \\
& \tan \theta\left[\mathfrak{R}\left(\lambda_{j}^{0}\right)+\sum_{k=1}^{N_{S}} \mathfrak{R}\left(r_{j k} G_{k}\left(\lambda_{j}^{0}\right)\right) \Delta x_{k 0}\right] \\
& \mathfrak{R}\left(\lambda_{j}^{0}\right)+\sum_{k=1}^{N_{s}} \mathfrak{R}\left(r_{j k} G_{k}\left(\lambda_{j}^{0}\right)\right) \Delta x_{k 0} \leq 0 .
\end{aligned}
$$

In the above equations, $\lambda_{j}^{0}$ denotes the current value of an eigenvalue with nonnegative imaginary part corresponding to a well damped mode. Equations (16) and (17) prohibit $\lambda_{j}$ from crossing the conic sector's upper and lower boundaries shown as solid lines in Fig. 1, whereas (18) keeps the real part of the eigenvalue negative.

\section{B. Sequential Conic Programming Algorithm}

The incremental eigenvalue shifting algorithm for PSS design solves a sequence of conic quadratic programs so that the eigenvalues of the unstable and poorly damped modes are shifted into the conic sector. Throughout the shifting process, the constraints in (16)-(18) are enforced. The eigenvalue shifting algorithm is described below.

Assume that $G_{k}(s)$ in (8) is specified. The user has to also choose the algorithm parameters: $\rho, \varsigma$, and $\varsigma^{\prime}$. Initially, $x_{k 0}=0$.

Step 1: Form the linear state-space equations for a given operating condition.

Step 2: Compute the system eigenvalues. The zero eigenvalues that are attributed to modeling assumptions are neglected.

$\underline{\text { Step 3: }}$ : Compute the damping ratio corresponding to each of the system modes. If all damping ratios are greater than $\varsigma$, print the solution and terminate the run.

Step 4: For each eigenvalue identified in step 2, compute the residues associated with the feedback of the PSS outputs to their inputs.

Step 5: Solve the conic quadratic program given by (10)-(18) to determine $\Delta x_{k 0}$ for $k=1, \ldots, N_{S}$.

Step 6: Update the current values of $x_{k 0}$ by the corresponding increments in step 5 .

Step 7: Update the state-space representation due to PSS feedback and go to step 2.

The above algorithm has been programmed in MATLAB. Some of the steps were implemented with the help of existing toolboxes or routines. Specifically, the computations in steps 1, 4 , and 7 are implemented via the Power System Toolbox [21] and its associated state-space objects. The conic program in step 5 is solved using SEDUMI [22].

It is interesting to note that unlike [5], [6], [9], [10], the above eigenvalue shifting algorithm does not require defining rectangular strips in the s-plane to constrain the locus of eigenvalue movement into the conic sector. As noted in [6], inappropriate choices of the amount of left eigenvalue shift and the corresponding width of the rectangular strip may result in an infeasible linear programming problem.

\section{Extension to Robust PSS Design}

The sequential conic approach for PSS design can be readily extended to account for multiple operating conditions. The resulting PSS design is termed robust because all system modes are guaranteed to remain well damped under each of the system operating conditions. Within the framework of the sequential conic programming approach, this would require minimizing the total sum of eigenvalue Euclidean distances (15) for all the operating cases, i.e.

$$
\operatorname{minimize} \sum_{c=1}^{N_{c}} \sum_{j=1}^{N_{a}^{c}} t_{j}^{c}
$$

where $c$ denotes the operating condition (case \#) and $N_{c}$ is the total number of cases. The feasible region for robust PSS design is formed of:

1. Constraints on the eigenvalue shifting of unstable and poorly damped modes (10)-(12) and (14) repeated for $c=1, \ldots, N_{c}$, i.e., all variables indexed with the eigenvalue number $j$ are re-indexed with both $j$ and the case number $c$.

2. Constraints restricting the eigenvalue movement of well damped modes (16)-(18) repeated for $c=1, \ldots, N_{c}$.

3. Constraints on the PSS incremental coefficient change (13).

The sequential conic programming approach would be applicable to robust PSS design with the understanding that the computations in steps $1-4$ and 7 are repeated for $c=1, \ldots, N_{c}$. The above conic program is solved in step 5.

\section{CoORdinated GAIN AND Phase Tuning}

Although the gain and phase compensation design approach is one of the most accepted techniques for PSS design, [9] and [10] have shown that there may be some benefit from the concurrent tuning of gain and phase. Reference [10] presents PSS designs corresponding to different operating conditions of the 39-bus New England test system, however, the different conditions are not considered simultaneously to yield a single robust PSS design. In fact, [6] shows that designing controllers for what may be perceived as the most stressed operating condition may result in unstable or poorly damped modes in other operating conditions. 
This section extends the sequential conic programming approach to the coordinated tuning of the phase compensation blocks and gains of the various PSSs under different operating conditions. The requirement in this case is to obtain the values of $\left(x_{k 0}, x_{k 1}, x_{k 2}\right)$ for $k=1, \ldots, N_{S}$ by using (6) instead of (7) in the conic programming implementation. The quadratic cone program for incremental eigenvalue shifting is therefore

$$
\operatorname{minimize} \sum_{c=1}^{N_{c}} \sum_{j=1}^{N_{a}^{c}} t_{j}^{c} \text { subject to }
$$

1. for $c=1, \ldots, N_{c}$ and $j=1, \ldots, N_{a}^{c}$

$$
\begin{aligned}
& t_{j}^{c} \geq \sqrt{\left(t_{R j}^{c}\right)^{2}+\left(t_{I j}^{c}\right)^{2}} \\
& t_{R j}^{c}=\Re\left(\lambda_{j c}^{*}-\lambda_{j c}^{0}\right)-\sum_{k=1}^{N_{s}} \sum_{i=0}^{2} \mathfrak{R}\left(r_{j k}^{c} H_{k i}\left(\lambda_{j c}^{0}\right)\right) \Delta x_{k i} \\
& t_{I j}^{c}=\mathfrak{I}\left(\lambda_{j c}^{*}-\lambda_{j c}^{0}\right)-\sum_{k=1}^{N_{S}} \sum_{i=0}^{2} \mathfrak{I}\left(r_{j k}^{c} H_{k i}\left(\lambda_{j c}^{0}\right)\right) \Delta x_{k i} \\
& \lambda_{j c}^{*}=-\alpha_{j c}^{*}+i^{\prime} \omega_{j c}^{*}, \alpha_{j c}^{*} \geq 0, \omega_{j c}^{*}=\tan \left(\cos ^{-1} \varsigma^{\prime}\right) \alpha_{j c}^{*} .
\end{aligned}
$$

2. for $c=1, \ldots, N_{c}$ and $j=1, \ldots, N_{b}^{c}$

$$
\begin{aligned}
& \mathfrak{I}\left(\lambda_{j c}^{0}\right)+ \sum_{k=1}^{N_{s}} \sum_{i=0}^{2} \mathfrak{I}\left(r_{j k}^{c} H_{k i}\left(\lambda_{j c}^{0}\right)\right) \Delta x_{k i} \leq \\
&-\tan \theta\left[\mathfrak{R}\left(\lambda_{j c}^{0}\right)+\sum_{k=1}^{N_{s}} \sum_{i=0}^{2} \mathfrak{R}\left(r_{j k}^{c} H_{k i}\left(\lambda_{j c}^{0}\right)\right) \Delta x_{k i}\right] \\
& \mathfrak{I}\left(\lambda_{j c}^{0}\right)+\sum_{k=1}^{N_{S}} \sum_{i=0}^{2} \mathfrak{I}\left(r_{j k}^{c} H_{k i}\left(\lambda_{j c}^{0}\right)\right) \Delta x_{k i} \geq \\
& \tan \theta\left[\mathfrak{R}\left(\lambda_{j c}^{0}\right)+\sum_{k=1}^{N_{S}} \sum_{i=0}^{2} \mathfrak{R}\left(r_{j k}^{c} H_{k i}\left(\lambda_{j c}^{0}\right)\right) \Delta x_{k i}\right] \\
& \mathfrak{R}\left(\lambda_{j c}^{0}\right)+\sum_{k=1}^{N_{S}} \sum_{i=0}^{2} \mathfrak{R}\left(r_{j k}^{c} H_{k i}\left(\lambda_{j c}^{0}\right)\right) \Delta x_{k i} \leq 0 .
\end{aligned}
$$

3. for $k=1, \ldots, N_{S}$ and $i=0,1,2$

$$
x_{k i}^{0}+\Delta x_{k i} \geq 0,\left|\Delta x_{k i}\right| \leq \rho \text {. }
$$

In addition to constraints (21)-(28), a PSS phase lead characteristic is included by restricting the locations of the compensator's zeros. Towards this end, define

$$
\begin{aligned}
& \bar{s}=s T_{k} \\
& \bar{x}_{k 1}=\frac{x_{k 1}}{x_{k 0} T_{k}} \\
& \bar{x}_{k 2}=\frac{x_{k 2}}{x_{k 0} T_{k}^{2}}
\end{aligned}
$$

then $F_{k}(s)$ in (1) can be written in normalized form with a double pole at $\bar{s}=-1$ :

$$
F_{k}(\bar{s})=x_{k 0} \frac{\bar{x}_{k 2} \bar{s}^{2}+\bar{x}_{k 1} \bar{s}+1}{(1+\bar{s})^{2}} .
$$

Following [9], assume that $F_{k}(s)$ is formed of two conventional lead-lag blocks such that the distance between each real zero-pole pair is at most one decade. This condition is equivalent to the following constraints on the real zeros of $F_{k}(\bar{s})$

$$
\begin{aligned}
& \frac{-\bar{x}_{k 1}+\sqrt{\bar{x}_{k 1}^{2}-4 \bar{x}_{k 2}}}{2 \bar{x}_{k 2}} \leq-0.1 \\
& \frac{-\bar{x}_{k 1}-\sqrt{\bar{x}_{k 1}^{2}-4 \bar{x}_{k 2}}}{2 \bar{x}_{k 2}} \geq-1 .
\end{aligned}
$$

The condition of the zeros being real is

$$
\bar{x}_{k 1} \geq 2 \sqrt{\bar{x}_{k 2}} \text {. }
$$

Equations (33) and (34) can be simplified into linear inequality constraints

$$
\begin{aligned}
& 10 \bar{x}_{k 1}-\bar{x}_{k 2} \leq 100 \\
& \bar{x}_{k 1}-\bar{x}_{k 2} \leq 1 .
\end{aligned}
$$

Constraints (35)-(37) define the non-convex feasible region bounded by the solid lines in Fig. 2. To yield a convex region, the right hand side of (35) is replaced by the equation of the tangent to the curve

$$
\bar{x}_{k 1} \geq \sqrt{\bar{x}_{k 2}^{t}}+\bar{x}_{k 2} / \sqrt{\bar{x}_{k 2}^{t}}
$$

where $\bar{x}_{k 2}^{t}$ is the abscissa at the point of tangency. In terms of the PSS incremental variables $\left(\Delta x_{k 0}, \Delta x_{k 1}, \Delta x_{k 2}\right)$, the feasible region constraints formed by (36)-(38) reduce to

$$
\begin{gathered}
-100 T_{k}^{2} \Delta x_{k 0}+10 T_{k} \Delta x_{k 1}-\Delta x_{k 2} \leq \\
100 T_{k}^{2} x_{k 0}^{0}-10 T_{k} x_{k 1}^{0}+x_{k 2}^{0} \\
-T_{k}^{2} \Delta x_{k 0}+T_{k} \Delta x_{k 1}-\Delta x_{k 2} \leq T_{k}^{2} x_{k 0}^{0}-T_{k} x_{k 1}^{0}+x_{k 2}^{0} \\
-\sqrt{\bar{x}_{k 2}^{t}} T_{k}^{2} \Delta x_{k 0}+T_{k} \Delta x_{k 1}-\Delta x_{k 2} / \sqrt{\bar{x}_{k 2}^{t}} \geq \\
\sqrt{\bar{x}_{k 2}^{t}} T_{k}^{2} x_{k 0}^{0}-T_{k} x_{k 1}^{0}+x_{k 2}^{0} / \sqrt{\bar{x}_{k 2}^{t}}
\end{gathered}
$$

Equation (41) is initialized with $\bar{x}_{k 2}^{t}=30.25$ so that the resulting triangular shaped region in Fig. 2 occupies the absolute maximum possible portion of the non-convex feasible region. However, as the PSS parameters are updated within the sequential algorithm, the tangency point is chosen as the current value of $\bar{x}_{k 2}$ given by (31).

The sequential conic programming algorithm for coordinated gain and phase tuning is summarized below. Initially, $x_{k 0}=x_{k 1}=x_{k 2}=0$ and $\bar{x}_{k 2}^{t}=30.25$.

Step 1: Form the linear state-space equations for each operating condition.

Step 2: Compute the system eigenvalues for each operating condition. The zero eigenvalues that are attributed to

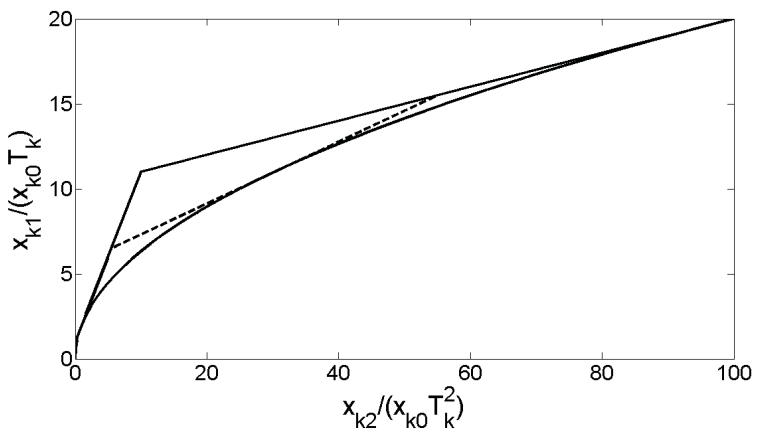

Fig. 2: Feasible region of PSS zeros. 
modeling assumptions are neglected.

Step 3: Compute the damping ratio corresponding to each of the system modes in all operating conditions. If all damping ratios are greater than $\varsigma$, print the solution and terminate the run.

Step 4: For each eigenvalue identified in step 2, compute the residues associated with the feedback of the PSS outputs to their inputs.

Step 5: Solve the conic quadratic program given by (20)-(28) and (39)-(41) to determine $\left(\Delta x_{k 0}, \Delta x_{k 1}, \Delta x_{k 2}\right)$ for $k=1, \ldots, N_{S}$. Step 6: Update the current values of $\left(x_{k 0}, x_{k 1}, x_{k 2}\right)$ by the corresponding increments in step 5 and set $\bar{x}_{k 2}^{t}=\bar{x}_{k 2}$.

Step 7: Update the state-space representation due to PSS feedback in each operating condition and go to step 2 .

\section{NUMERICAL RESULTS}

Numerical testing is carried out on a 16 machine, 68-bus test system which is a reduced order equivalent of the interconnected New England Test System (NETS) and the New York Power System (NYPS). This test system consists of five geographical areas, two of which are represented by generator groups in the NETS and the NYPS, with the remaining three represented by equivalent generator models. There are three transfer corridors between NETS and NYPS connecting buses \#60-\#61, \#53-\#54, and \#27-\#53. Each of the corridors has double circuit tie-lines and the tie-line power exchange between the NETS and the NYPS is $700 \mathrm{MW}$ at steady-state. The machines are represented by the sub-transient model. The power flow and machine dynamic data are given in [17]. Each generator is assumed to be equipped with a power system stabilizer and a static excitation system with a time constant $T_{a}=0.05 \mathrm{~s}$ and a moderate gain given by $T_{d 0}^{\prime} / 2 T_{a}$ [8], [10], where $T_{d 0}^{\prime}$ is the generator $\mathrm{d}$-axis open-circuit transient time constant.

The program parameters utilized in the test cases are $\rho=1$ (trust-region parameter) and $\varsigma^{\prime}=1.1 \varsigma$ (target damping ratio). The (boundary) damping ratio $\varsigma$ takes the values $5 \%$, $10 \%$, and $15 \%$. Table I shows 9 operating conditions in the model bank, eight of which appear in [17]. Case \#1 is the base case with $700 \mathrm{MW}$ power flow through the tie-lines and a constant impedance (CI) load model. Cases \#2 through \#5 model the outage of one of the tie-lines between buses \#53$\# 54$, \#60-\#61, and \#27-\#53, respectively. Cases \#6 and \#7 correspond to a change in the tie-line flows. Cases \#7 and \#8 are combinations of constant current $(\mathrm{CC})$, constant power (CP), and CI load models. Case \#9 represents the outage of two tie-lines, one between buses \#53-\#54 and the other between buses \#60-\#61. Fig. 3 shows a map of the open loop eigenvalues for the nine operating conditions in Table I. Only the eigenvalues having their real part greater than -3.5 are shown. The figure also shows the $5 \%, 10 \%$, and $15 \%$ damping ratio lines.

The 16 power system stabilizers are assumed to initially have typical speed measurement parameters with a washout time constant of $10 \mathrm{~s}$ and one decade between the real zeropole pair:

$$
H_{k}(s)=x_{k 0} \frac{(1+0.1 s)^{2}}{(1+0.01 s)^{2}} \frac{10 s}{1+10 s}, k=1, \ldots, 16 .
$$

TABLE I

OPERATING CONDITIONS USED IN THE MODEL BANK

\begin{tabular}{|l|l|l|l|}
\hline $\begin{array}{c}\text { Case } \\
\#\end{array}$ & $\begin{array}{c}\text { Tie-Line } \\
\text { Flow }(\mathbf{M W})\end{array}$ & Outage of Line & Type of Load \\
\hline 1 & 700 & no outage & CI \\
\hline 2 & 700 & $53-54$ & CI \\
\hline 3 & 700 & $60-61$ & CI \\
\hline 4 & 700 & $27-53$ & CI \\
\hline 5 & 100 & no outage & CI \\
\hline 6 & 900 & no outage & CI \\
\hline 7 & 700 & no outage & $50 \%$ CI \& 50\% CC \\
\hline 8 & 700 & no outage & $50 \%$ CI \& 50\% CP \\
\hline 9 & 700 & $53-54 \& 60-61$ & CI \\
\hline
\end{tabular}

TABLE II

COORDINATED GAIN TUNING RESUlts: $x_{k 0}$

\begin{tabular}{|c|c|c|c|c|}
\hline \multirow{2}{*}{ Gen. \# $\boldsymbol{k}$} & \multicolumn{4}{|c|}{ Damping Ratio } \\
\cline { 2 - 5 } & $\mathbf{5 \%}$ & $\mathbf{1 0 \%}$ & $\mathbf{1 5 \%}$ & $\mathbf{5 \% *}$ \\
\hline 1 & 5.9215 & 11.0000 & 12.0000 & 8.4391 \\
\hline 2 & 5.7433 & 11.0000 & 20.0000 & 8.1869 \\
\hline 3 & 8.4054 & 11.0000 & 20.0000 & 8.2405 \\
\hline 4 & 5.6164 & 8.9814 & 18.0000 & 10.4377 \\
\hline 5 & 2.4691 & 5.9285 & 18.0000 & - \\
\hline 6 & 6.5232 & 9.0000 & 17.1509 & 10.4231 \\
\hline 7 & 6.6665 & 9.0000 & 16.0000 & 10.4314 \\
\hline 8 & 5.1740 & 7.0000 & 7.7852 & 9.7371 \\
\hline 9 & 8.3673 & 10.7715 & 20.0000 & 8.5032 \\
\hline 10 & 5.5730 & 11.0000 & 14.0000 & 9.7994 \\
\hline 11 & 5.5812 & 10.7487 & 16.0000 & 6.2819 \\
\hline 12 & 6.5455 & 11.0000 & 14.6971 & 5.8405 \\
\hline 13 & 4.7390 & 11.0000 & 20.0000 & 5.5037 \\
\hline 14 & 4.7813 & 11.0000 & 20.0000 & 10.6409 \\
\hline 15 & 0.0000 & 11.0000 & 20.0000 & - \\
\hline 16 & 3.8643 & 11.0000 & 20.0000 & - \\
\hline
\end{tabular}

TABLE III

CoORdinated Gain AND Phase TUNING Results - Damping Ratio $=10 \%$

\begin{tabular}{|c|c|c|c|c|c|}
\hline Gen. \# $\boldsymbol{k}$ & $\boldsymbol{x}_{\boldsymbol{k} 0}$ & $\boldsymbol{x}_{\boldsymbol{k} 1}$ & $\boldsymbol{x}_{\boldsymbol{k} 2}$ & $\boldsymbol{T}_{\boldsymbol{k} 1}(\mathrm{~s})$ & $\boldsymbol{T}_{\boldsymbol{k} 3}(\mathrm{~s})$ \\
\hline 1 & 10.9994 & 2.1999 & 0.1100 & 0.1000 & 0.1000 \\
\hline 2 & 10.9998 & 2.2000 & 0.1100 & 0.1000 & 0.1000 \\
\hline 3 & 10.9998 & 2.2000 & 0.1100 & 0.1000 & 0.1000 \\
\hline 4 & 6.9996 & 1.3999 & 0.0700 & 0.1000 & 0.1000 \\
\hline 5 & 5.6675 & 0.9106 & 0.0344 & 0.1000 & 0.0607 \\
\hline 6 & 8.9996 & 1.7999 & 0.0900 & 0.1000 & 0.1000 \\
\hline 7 & 8.9995 & 1.4932 & 0.0593 & 0.1000 & 0.0659 \\
\hline 8 & 8.1203 & 1.6241 & 0.0812 & 0.1000 & 0.1000 \\
\hline 9 & 10.9998 & 2.2000 & 0.1100 & 0.1000 & 0.1000 \\
\hline 10 & 10.9998 & 2.2000 & 0.1100 & 0.1000 & 0.1000 \\
\hline 11 & 10.9973 & 2.1995 & 0.1100 & 0.1000 & 0.1000 \\
\hline 12 & 10.9997 & 2.1999 & 0.1100 & 0.1000 & 0.1000 \\
\hline 13 & 10.9992 & 2.1998 & 0.1100 & 0.1000 & 0.1000 \\
\hline 14 & 10.9992 & 2.1998 & 0.1100 & 0.1000 & 0.1000 \\
\hline 15 & 10.9989 & 2.1998 & 0.1100 & 0.1000 & 0.1000 \\
\hline 16 & 10.9994 & 2.1999 & 0.1100 & 0.1000 & 0.1000 \\
\hline
\end{tabular}


The coordinated gain tuning approach in section IV is used with three values of the damping ratio to yield the PSS gains in Table II. It is interesting to note that for a damping ratio of $5 \%$, the program chooses not to have a PSS installed on machine \#15 and assigns very low gains for the PSSs at machines \#5 and \#16. A more practical solution of coordinated gain tuning is given in the rightmost column of Table II (marked with a star sign) where the machines \#5, \#15, and \#16 are not equipped with PSSs. Fig. 3 also shows the corresponding closed loop eigenvalues where it is seen that the $15 \%$ damping ratio is achieved for all operating cases. For illustration, Fig. 4 shows the generalized root locus for case $\# 1$. The root locus is traced by the increase of the PSS gains as given by the conic quadratic program solution, from zero up to their coordinated design values. It demonstrates the agreement in the values of the points on the locus obtained from the sensitivity equation (7) and from direct eigenvalue computation of the updated state-space representation. This agreement has been also observed in the loci of cases \#2 through \#9 and is expected because of the use of the trustregion technique. The design exercise was repeated with $\left(x_{k 0}, x_{k 1}, x_{k 2}\right)$ treated as variables for $k=1, \ldots, 16$. In this design, the known PSS parameters in (1) are assumed to be $T_{w}=10 \mathrm{~s}$ and $T_{k}=0.01 \mathrm{~s}$. The sequential conic programming approach of section $\mathrm{V}$ gives the closed loop eigenvalue map in Fig. 5. Table III shows the PSS design parameters and lead time constants for a damping ratio of $10 \%$. It is important to note that in practice no more than two digits are needed for the parameter values in Tables II and III. The corresponding generalized root locus for case \#1 is shown in Fig. 6 and also validates the accuracy of the locus following method with three variable parameters per PSS.

In practice, any linear stabilizer design needs to be checked for robustness under different operating conditions by using a transient stability simulation. The results of two simulations, each executed for $30 \mathrm{~s}$, are presented herein for the PSS design in Table III. In simulation \#1, one of the tie-lines between buses \#60-\#61 is assumed to be out of service and a threephase fault at bus \#53 is simulated for $70 \mathrm{~ms}$ in one of the tielines between bus \#53 and bus \#54. The fault is cleared by the removal of the faulted circuit. The variations of the machine angles are computed with respect to machine \#15 [11]. The relative angle variation of one machine from each group is plotted in Fig. 7 for the first $10 \mathrm{~s}$ of the simulation. It is clear that the oscillations settle down due to the PSS action. Simulation \#2 models a $5 \%$ step increase in the voltage reference of the excitation system of machine \#1. The corresponding angular variation plots are shown in Fig. 8. In both simulations, the upper and lower output limits of each power system stabilizer are set to $0.15 \mathrm{pu}$ and $-0.1 \mathrm{pu}$, respectively. For illustration, Figure 9 shows the output of the PSSs at machines \#1, \#13, \#14, and \#16 in simulation \#1.

To investigate the possible advantage of coordinated gain and phase tuning as opposed to the coordinated gain tuning method, measures of the control effort and performance are compared for each of the two above time-domain simulations. The control effort is measured in terms of the PSS output $u_{k}(t)$ as [12]

$$
C E=\sum_{k=1}^{16} \int_{t=0}^{t=30 \mathrm{~s}}\left(u_{k}(t)\right)^{2} d t .
$$

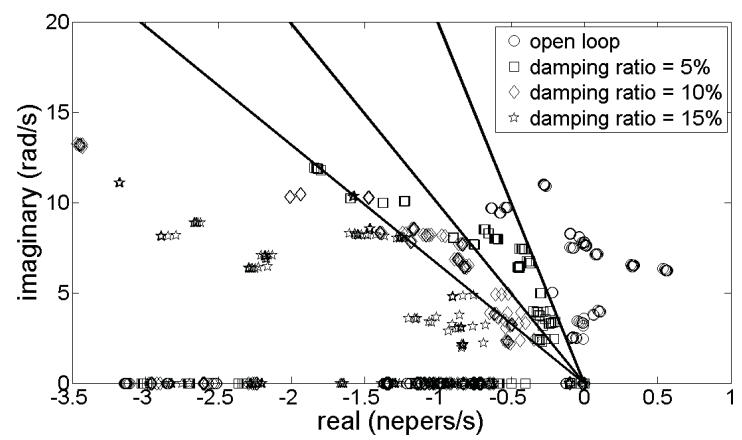

Fig. 3: Open- and closed-loop poles for 9 operating conditions - coordinated gain tuning.

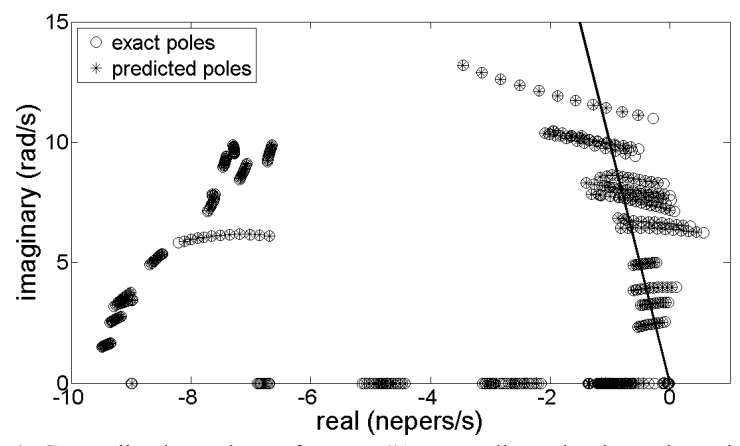

Fig. 4: Generalized root locus for case \#1 - coordinated gain tuning with $10 \%$ damping ratio.

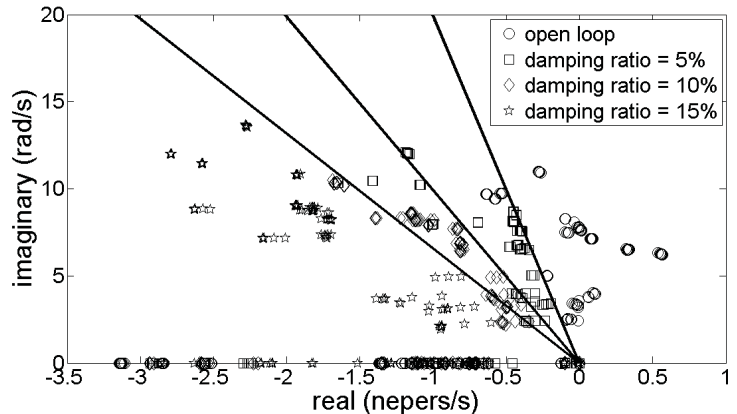

Fig. 5: Open- and closed-loop poles for 9 operating conditions - coordinated gain and phase tuning.

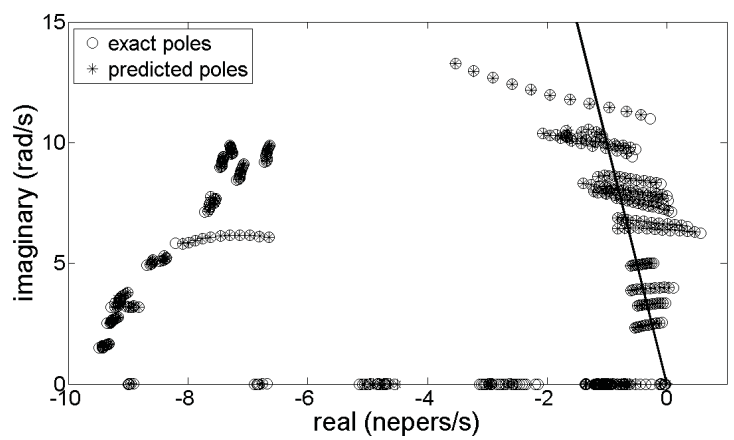

Fig. 6: Generalized root locus for case \#1 - coordinated gain and phase tuning with $10 \%$ damping ratio. 

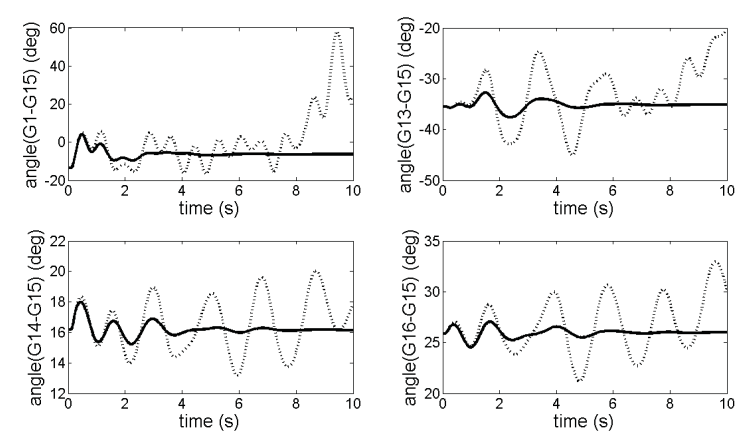

Fig. 7: Dynamic response of the system in simulation \#1 - solid line: with PSS, dotted line: without PSS.
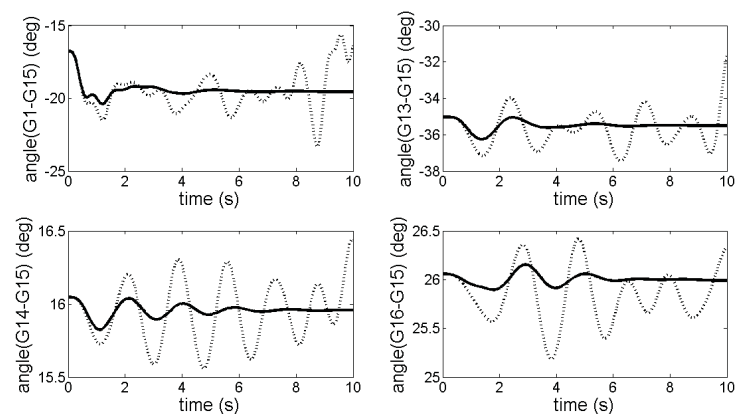

Fig. 8: Dynamic response of the system in simulation $\# 2$ - solid line: with PSS, dotted line: without PSS.
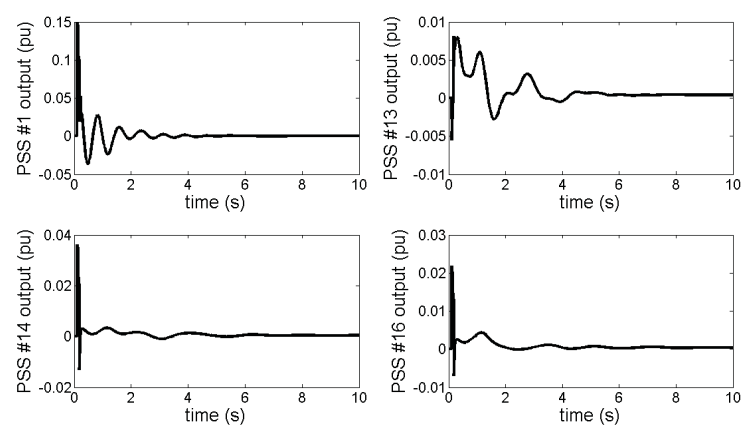

Fig. 9: Dynamic output of PSSs in simulation \#1.

The performance is gauged by two indices that reflect the settling time $\left(P I_{1}\right)$ and overshoot $\left(P I_{2}\right)$ as a function of the machine speed deviation $\Delta \omega_{k}(t)[16]$

$$
\begin{aligned}
& P I_{1}=\sum_{k=1}^{16} \int_{t=0}^{t=30 s}\left(t \Delta \omega_{k}(t)\right)^{2} d t \\
& P I_{2}=\sum_{k=1}^{16} \int_{t=0}^{t=30 s}\left(\Delta \omega_{k}(t)\right)^{2} d t .
\end{aligned}
$$

The values of the indices (43)-(45) are shown in Tables IV and $\mathrm{V}$ for simulations $\# 1$ and $\# 2$, respectively. The results suggest that both methods perform well in terms of damping oscillations with comparable control effort. This is explained by the suitability of the chosen phase characteristic in the coordinated gain tuning approach.

Table VI shows the computational effort of the sequential conic programming approach, i.e. the number of eigenvalue
TABLE IV

\begin{tabular}{|c|c|c|c|c|c|c|}
\hline \multirow[t]{2}{*}{$\begin{array}{l}\text { Damp. } \\
\text { Ratio }\end{array}$} & \multicolumn{3}{|c|}{$\begin{array}{c}\text { Coordinated Gain } \\
\text { Tuning } \\
\end{array}$} & \multicolumn{3}{|c|}{$\begin{array}{c}\text { Coordinated Gain \& Phase } \\
\text { Tuning }\end{array}$} \\
\hline & $C E$ & $P I_{1}$ & $P I_{2}$ & $C E$ & $P I_{1}$ & $\mathrm{PI}_{2}$ \\
\hline w/o PSS & - & 3.38 & $2.12 \mathrm{E} 3$ & - & 3.378 & $2.12 \mathrm{E} 3$ \\
\hline $5 \%$ & $5.47 \mathrm{E}-3$ & $5.52 \mathrm{E}-5$ & $8.11 \mathrm{E}-3$ & $4.04 \mathrm{E}-3$ & $5.45 \mathrm{E}-5$ & $6.70 \mathrm{E}-3$ \\
\hline $10 \%$ & $1.24 \mathrm{E}-2$ & $3.26 \mathrm{E}-5$ & $3.46 \mathrm{E}-3$ & $1.24 \mathrm{E}-2$ & $3.28 \mathrm{E}-5$ & $3.47 \mathrm{E}-3$ \\
\hline $15 \%$ & $2.11 \mathrm{E}-2$ & $2.35 \mathrm{E}-5$ & $1.66 \mathrm{E}-3$ & $2.09 \mathrm{E}-2$ & $2.25 \mathrm{E}-5$ & $1.47 \mathrm{E}-3$ \\
\hline
\end{tabular}

CONTROL EFFORT\& PERFORMANCE INDICES - SIMULATION \#1

TABLE V

CONTROL EFFORT\& PERFORMANCE INDICES - SIMULATION \#2

\begin{tabular}{|c|c|c|c|c|c|c|}
\hline \multirow{2}{*}{$\begin{array}{c}\text { Damp. } \\
\text { Ratio }\end{array}$} & \multicolumn{3}{|c|}{$\begin{array}{c}\text { Coordinated Gain } \\
\text { Tuning }\end{array}$} & \multicolumn{3}{c|}{ Coordinated Gain \& Phase } \\
& $\boldsymbol{C E}$ & $\boldsymbol{P I}_{\mathbf{1}}$ & $\boldsymbol{P I}_{\mathbf{2}}$ & $\boldsymbol{C E}$ & $\boldsymbol{P I}_{\boldsymbol{1}}$ & $\boldsymbol{P I}_{\boldsymbol{2}}$ \\
\cline { 2 - 7 } & - & 1.19 & $6.49 \mathrm{E} 2$ & - & 1.19 & $6.49 \mathrm{E} 2$ \\
\hline w/o PSS & - & $8.16 \mathrm{E}-5$ & $3.60 \mathrm{E}-2$ & $3.99 \mathrm{E}-4$ & $6.67 \mathrm{E}-5$ & $2.95 \mathrm{E}-2$ \\
\hline $5 \%$ & $3.93 \mathrm{E}-4$ & $2.16 \mathrm{E}-5$ & $1.42 \mathrm{E}-2$ & $4.83 \mathrm{E}-4$ & $3.17 \mathrm{E}-5$ & $1.43 \mathrm{E}-2$ \\
\hline $10 \%$ & $4.84 \mathrm{E}-4$ & 3.12 & & \\
\hline $15 \%$ & $6.36 \mathrm{E}-4$ & $1.40 \mathrm{E}-5$ & $6.36 \mathrm{E}-3$ & $7.03 \mathrm{E}-4$ & $1.21 \mathrm{E}-5$ & $5.53 \mathrm{E}-3$ \\
\hline
\end{tabular}

TABLE VI

COMPARING COMPUtational PERFormances of GAIN AND PHASE-GAIN COORDINATED AND ROBUST PSS DESIGN

\begin{tabular}{|c|c|c|c|c|c|c|}
\hline \multirow{2}{*}{$\begin{array}{l}\text { Damp. } \\
\text { Ratio }\end{array}$} & \multicolumn{3}{|c|}{$\begin{array}{c}\text { Coordinated Gain } \\
\text { Tuning }\end{array}$} & \multicolumn{2}{c|}{$\begin{array}{c}\text { Coordinated Gain \& Phase } \\
\text { Tuning }\end{array}$} \\
\cline { 2 - 4 } & \multirow{2}{*}{ iter. } & \multicolumn{2}{|c|}{ time (s) } & \multirow{2}{*}{ iter. } & \multicolumn{2}{|c|}{ time (s) } \\
\cline { 3 - 4 } \cline { 5 - 6 } & & conic & total & & conic & total \\
\hline $5 \%$ & 10 & 30 & 99 & 9 & 301 & 373 \\
\hline $10 \%$ & 12 & 34 & 120 & 12 & 278 & 386 \\
\hline $15 \%$ & 21 & 61 & 211 & 25 & 631 & 845 \\
\hline
\end{tabular}

shifting iterations (iter.), the computational time for solving the conic programs, and the total computation time. In all cases, the iterations are less than 25 and are completed within 4 minutes for coordinated gain tuning and 14 minutes for phasegain coordinated tuning. The computational times were recorded on an Intel ${ }^{\circledR}$ Core $^{\mathrm{TM}} 2$ Duo Processor T5300 (1.73 GHz) PC with 1 GB RAM. By increasing the trust-region limit $\rho$, the sequential conic programming approach would require a lower number of eigenvalue shifting iterations. However, a large value of $\rho$ would compromise the accuracy of the eigenvalue shift given by (6) and (7).

The coordinated PSS tuning method can be employed for the stabilization of large-scale power systems. In this case, the computation of eigenvalues would require the use of sparse eigensolution algorithms [23], [24].

\section{CONCLUSION}

This paper presented a sequential conic programming implementation of a multivariable root locus following technique for robust PSS design. A trust-region is employed to ensure the validity of the conic programming solution. This solution directs the locus of eigenvalues corresponding to unstable and poorly damped modes to a satisfactory region in the s-plane. The technique is demonstrated on problems involving coordinated gain tuning and coordinated gain and phase tuning with different operating scenarios. An important conclusion of this work is that the coordinated gain strategy, in which the phase compensation is carried out individually and in a previous stage, produces solutions that are practically as good as those obtained by coordinated gain and phase tuning. 
This conclusion comes in support of the claims made by [8] that coordinated gain tuning usually suffices for practical power system applications. The sequential conic programming approach is very generic. It can be easily extended to design robust damping by FACTS devices such as static VAr compensators and thyristor controlled series capacitors. We are currently working on sequential conic programming based coordinated design of PSSs and FACTS devices to effectively damp local and inter-area modes. Application to large scale power system models will follow next with the use of sparse eigensolution methods and state of the art conic solvers which have been made recently available.

\section{ACKNOWLEDGEMENT}

The authors would like to thank Prof. Graham Rogers, Cherry Tree Scientific Software, for providing access to the power systems toolbox (PST).

\section{REFERENCES}

[1] G. Rogers, Power System Oscillations. Norwell: Kluwer Academic Publishers, 2000.

[2] E. V. Larsen and D. A. Swann, "Applying power system stabilizers part II: performance objectives and tuning concepts," IEEE Trans. Power App. Syst., vol. PAS-100, no. 6, pp. 3025-3033, June 1981.

[3] P. Kundur, M. Klein, G. J. Rogers, and M. S. Zywno, "Application of power system stabilizers for enhancement of overall system stability," IEEE Trans. Power Syst., vol. 4, no. 2, pp. 614-626, May 1989.

[4] M. J. Gibbard, "Robust design of fixed-parameter power system stabilizers over a wide range of operating conditions," IEEE Trans. Power Syst., vol. 6, no. 2, pp. 794-800, May 1991.

[5] A. Doi and S. Abe, "Coordinated synthesis of power system stabilizers in multimachine power systems," IEEE Trans. Power App. Syst., vol. PAS-103, no. 6, pp. 1473-1479, June 1984.

[6] P. Pourbeik and M. J. Gibbard, "Simultaneous coordination of power system stabilizers and FACTS device stabilizers in a multimachine power system for enhancing dynamic performance," IEEE Trans. Power Syst., vol. 13, no. 2, pp. 473-479, May 1998.

[7] J. C. R. Ferraz, N. Martins, and G. N. Taranto, "Simultaneous partial pole placement for power system oscillation damping control," IEEE Power Engineering Society Winter Meeting, vol. 3, pp. 1154-1159, 28 Jan.-1 Feb. 2001.

[8] J. C. R. Ferraz, N. Martins, and G. N. Taranto, "Coordinated stabilizer tuning in large power systems considering multiple operating conditions," IEEE Power Engineering Society General Meeting, pp. 18, 24-28 June 2007

[9] J. J. Da Cruz and L. C. Zanetta Jr., "Stabilizer design for multimachine power systems using mathematical programming," Elec. Power Energy Syst., vol. 19, no. 8, pp. 519-523, 1997.

[10] L. C. Zanetta Jr. and J. J. Da Cruz, "An incremental approach to the coordinated tuning of power system stabilizers using mathematical programming," IEEE Trans. Power Syst., vol. 20, no. 2, pp. 895-902, May 2005.

[11] B. C. Pal, A. H. Coonick, and D. C. Macdonald, "Robust damping controller design in power systems with superconducting magnetic energy storage devices," IEEE Trans. Power Syst., vol. 15, no. 1, pp. 320-325, Feb. 2000

[12] B. C. Pal, "Robust pole placement versus root-locus approach in the context of damping interarea oscillations in power systems," IEE Proc.Gener. Transm. Dstrib., vol. 149, no. 6, pp. 739-745, Nov. 2002.

[13] B. C. Pal, A. H. Coonick, I. M. Jaimoukha, and H. El-Zobaidi, "A linear matrix inequality approach to robust damping control design in power systems with superconducting magnetic energy storage device," IEEE Trans. Power Syst., vol. 15, no. 1, pp. 356-362, Feb. 2000.

[14] A. L. B. do Bomfim, G. N. Taranto, and D. M. Falcão, "Simultaneous tuning of power system damping controllers using genetic algorithms," IEEE Trans. Power Syst., vol. 15, no. 1, pp. 163-169, Feb. 2000.

[15] P. Zhang and A. H. Coonick, "Coordinated synthesis of PSS parameters in multi-machine power systems using the method of inequalities applied to genetic algorithms," IEEE Trans. Power Syst., vol. 15, no. 2, pp. 811-816, May 2000

[16] Y. L. Abdel-Magid and M. A. Abido, "Optimal multiobjective design of robust power system stabilizers using genetic algorithms," IEEE Trans. Power Syst., vol. 18, no. 3, pp. 1125-1132, Aug. 2003

[17] B. Pal and B. Chaudhuri, Robust Control in Power Systems. NY: Springer, 2005

[18] A. Ben-Tal and A. Nemirovski, Lectures on Modern Convex Optimization. SIAM, 2001.

[19] X.-M. Xu, Z.-M. Wu, and Z.-J. Zhang, "A design method of generalized root-loci for MIMO systems," $25^{\text {th }}$ IEEE Conference on Decision and Control, vol. 25, no. 1, pp. 713-717, Dec. 1986.

[20] J. R. Mitchell and W. L. McDaniel Jr., "A generalized root locus following technique," IEEE Trans. Automatic Control, vol. 15, no. 4, pp. 483-486, Aug. 1970.

[21] Power System Toolbox Vesion 3.0. Copyright Joe Chow/Graham Rogers 1991-2008.

[22] J. F. Sturm, "Using SeDuMi 1.02, a Matlab toolbox for optimization over symmetric cones," Optimization Methods and Software, vol. 1112, pp. 625-653, 1999.

[23] J. Rommes and N. Martins, "Efficient computation of transfer function dominant poles using subspace acceleration," IEEE Trans. Power Syst., vol. 21, no. 3, pp. 1218-1226, Aug. 2006.

[24] J. Rommes and N. Martins, "Efficient computation of multivariable transfer function dominant poles using subspace acceleration," IEEE Trans. Power Syst., vol. 21, no. 4, pp. 1471-1483, Nov. 2006

Rabih A. Jabr (M' 02) was born in Lebanon. He received the B.E. degree in electrical engineering (with high distinction) from the American University of Beirut, Beirut, Lebanon, in 1997 and the Ph.D. degree in electrical engineering from Imperial College London, London, U.K., in 2000.

Currently, he is an Associate Professor and serves as the chairperson of the Electrical, Computer and Communication Engineering Department at Notre Dame University, Lebanon. His research interests are in mathematical optimization techniques, design optimization, optimal power flow, and state estimation

Bikash C. Pal (M'00-SM'02) received the B.E.E. (with honors) degree from Jadavpur University, Calcutta, India, the M.E. degree from the Indian Institute of Science, Bangalore, India, and the Ph.D. degree from Imperial College London, London, U.K., in 1990, 1992, and 1999, respectively, all in electrical engineering.

Currently, he is a Reader in the Department of Electrical and Electronic Engineering, Imperial College London. His current research interests include state estimation, power system dynamics, and flexible ac transmission system controllers.

Nelson Martins (F'98) received the B.Sc. degree in electrical engineering from University of Brasilia, Brasilia, Brazil, and the M.Sc. and Ph.D. degrees in electrical engineering from the University of Manchester Institute of Science and Technology (UMIST), Manchester, U.K., in 1972, 1974, and 1978 , respectively.

Since 1978, he has been with CEPEL, Rio de Janeiro, Brazil, developing computer tools for power system dynamics and control and specialized eigensolution methods. 